\title{
Toward a Scientific and Engineering Discipline of Cyber-Physical Systems
}

Cyber-physical systems (CPS) are driving a wide range of exciting applications from smart cities to smart healthcare. In contrast to traditional embedded systems, CPS operate in unpredictable environments in which they must meet stringent requirements such as end-to-end timeliness and physical system stability. The field of CPS addresses these critical challenges through seamless integration of computing and physical components. In the second decade since its inception as an interdisciplinary field, CPS is growing as a vibrant scientific and engineering discipline.

I am honored to be named the new editor-in-chief of ACM Transactions on Cyber-Physical Systems (TCPS). TCPS has established itself as a leading journal in the field of CPS under the great leadership of Tei-Wei Kuo as the founding editor-in-chief. While traditional computer science disciplines are dominated by their premier conferences, the diverse and interdisciplinary nature of CPS provides a unique opportunity for TCPS to become the premier venue for publishing CPS research. Our aspiration is to grow TCPS into the flagship publication where best CPS works are published in a timely fashion, covering both the foundation and emergent frontiers of CPS research. In the following I'd like to share some initiatives that the editorial board is undertaking to realize our aspiration.

Special issues on emerging topics. Special issues have been instrumental for establishing the topics and growing the submission pipeline of TCPS. Given the rapid evolution of CPS, we will continue to organize special issues on emerging topics. New topics on CPS often face challenges at established publication venues due to their interdisciplinary nature. TCPS aims to fill the gap with special issues that capture the state of the art of the new topics and shape the evolving areas. The success of special issues depends on the impacts of the topics and the leadership of the guest editors. We welcome strong proposals from the community on emerging topics of CPS.

Foundation and core technologies. In parallel to the development of innovative applications and point solutions, we are witnessing the emergence of the foundation and core technologies of CPS as a scientific and engineering discipline. Examples range from cyber-physical co-design approaches to holistic system architectures crosscutting cyber and physical components. We welcome research papers on foundations and core CPS technologies underpinning the field of CPS. We will balance the special issues and regular issues to cover both new and established topics. Furthermore, some of the special issues will help evolve new topics toward established areas of CPS that continue to attract regular submissions.

Timely, predictable, and rigorous reviews. Lengthy and unpredictable review cycles are major factors that discourage authors from submitting to journals. We aim to streamline the review process based on the best practices of ACM publications. Furthermore, we plan to incorporate and adapt well tested approaches of premier conferences to shorten review cycles. A timely, predictable, and rigorous review process will attract strong submissions to TCPS, which will in

\footnotetext{
ACM Reference format:

https://doi.org/10.1145/3464945

(c) 2021 Copyright held by the owner/author(s).

2378-962X/2021/07-ART22e

https://doi.org/10.1145/3464945
}

Chenyang Lu. 2021. Toward a Scientific and Engineering Discipline of Cyber-Physical Systems. ACM Trans. Cyber-Phys. Syst. 5, 3, Article 22e (July 2021), 2 pages. 
turn contribute to the field of CPS by providing a publication with high-quality and cutting-edge articles.

CPS is a vibrant interdisciplinary field that continues to open new grounds while establishing its core and foundation. I look forward to working with the outstanding editorial board and the CPS community to make TCPS the flagship journal for the field of CPS. Through our collective effort, TCPS will play an instrumental role in establishing and growing the scientific and engineering discipline of CPS.

Chenyang Lu

Editor-in-Chief ACM Transactions on Cyber-Physical Systems 